\title{
DESARROLLO DE UN MÉTODO DE CALIBRACIÓN DE VELOCÍMETROS EN SU FUNCIÓN DE MEDICIÓN DE DISTANCIA, ESTIMACIÓN DE SU INCERTIDUMBRE Y VALIDACIÓN
}

Raziel Farid Sanabria Sandí

\section{Resumen}

Los velocímetros son utilizados para la comprobación de odómetros y para la verificación de taxímetros en líneas de inspección vehicular y talleres de servicio mecánico para automóviles. En la Universidad de Costa Rica se desarrolló un procedimiento de calibración de estos equipos en su función de medición de distancia.

Se presenta el desarrollo del modelo de medida completo, así como el detalle de la estimación de su respectiva incertidumbre considerando todas las posibles fuentes que afectan en forma relevante a la medición.

Además, se presentan los resultados de la validación, en la cual se demuestra que el método de calibración desarrollado es capaz de medir el error de indicación de un equipo con una incertidumbre menor al 0,22 \% de la indicación del velocímetro.

Palabras clave: velocímetro, calibración, validación, metrología, incertidumbre.

\begin{abstract}
The speedometers are used for checking and verifying odometers in vehicle inspection lines and mechanical service shops for automobiles. A procedure for calibration of speedometers in their distance measurement function was developed at the University of Costa Rica.

It is presented the measurement model development as well as details of the estimation of its respective uncertainty considering all possible sources affecting relevantly to the measurement.

Furthermore, validation results are presented in which the calibration method developed is capable of measuring the error of this equipment with an uncertainty less than $0,22 \%$ of the indication of the speedometer.
\end{abstract}

Keywords: speedometer, calibration method, metrology, uncertainty.

Recibido: 24 del marzo del 2009 • Aprobado: 26 del mayo del 2009

\section{DEFINICIONES}

Análisis de varianza de un solo factor: técnica estadística utilizada para la prueba de homogeneidad de medias poblacionales para varias muestras.

Circómetro o cinta pi: cinta con un nonio utilizada para medir ya sea el contorno o diámetro de un cilindro en forma directa.

Coeficiente de rodadura: constante de proporcionalidad entre la fuerza de oposición a la rodadura de la(s) llantas(s) y el peso aplicado sobre las mismas.

Ecuación de Welch-Satterhwaite: ecuación utilizada para determinar el número efectivo de grados de libertad asociados a una incertidumbre estándar combinada.

Función de medición: función de magnitudes cuyo valor es un valor medido de la magnitud de salida en el modelo de medición, cuando se calcula mediante los valores conocidos de las magnitudes de entrada en el modelo de medición. Si el modelo de medición $h\left(Y, X_{l}, \ldots, X_{n}\right)=0$ puede escribirse explícitamente como $Y=f\left(X_{1}, \ldots, X_{n}\right)$, siendo $Y$ la magnitud de salida en el modelo de medición, $f$ es la función de medición. En general, $f$ puede representar un algoritmo que, para los valores de entrada $x_{1}, \ldots, x_{n}$, da como resultado un valor único de la magnitud de salida $y=f\left(x_{1}, \ldots, x_{n}\right)$. 
Incertidumbre estándar: incertidumbre de medida expresada como una desviación típica. Para una función de probabilidad $y=p(x)$ la incertidumbre estándar se define como

$$
\sqrt{\int_{-\infty}^{+\infty}(x-\bar{x})^{2} \cdot p(x) d x}
$$

Incertidumbre expandida: producto de la incertidumbre estándar combinada por el factor de cobertura, el cual depende del nivel de confianza.

Mejor estimador: valor de un parámetro estadístico que permite resumir la mayor información del parámetro contenido en la muestra.

Mensurando: magnitud que se desea medir. La especificación de un mensurando requiere el conocimiento de la naturaleza de la magnitud y la descripción del estado del fenómeno, cuerpo o sustancia cuya magnitud es una propiedad, incluyendo las componentes pertinentes y las entidades químicas involucradas.

Modelo de medición: relación matemática entre todas las magnitudes conocidas que intervienen en una medición. Una forma general del modelo de medición es la ecuación $h\left(Y, X_{1}, \ldots, X_{n}\right)=0$, donde $\mathrm{Y}$, la magnitud de salida del modelo de medición, es el mensurando, cuyo valor debe deducirse a partir de la información sobre las magnitudes de entrada en el modelo de medición $X_{1}, \ldots, X_{n}$.
Principio de medida: fenómeno de naturaleza física, química o biológica que sirve como base para una medición.

Sistema de medida: conjunto de uno o más instrumentos de medida $\mathrm{y}$, frecuentemente otros dispositivos, incluyendo reactivos e insumos varios, ensamblados y adaptados para proporcionar valores medidos dentro de los intervalos especificados, para magnitudes de naturalezas dadas. Un sistema de medición puede estar conformado por un único instrumento de medida.

Trazabilidad metrológica: propiedad de un resultado de medida por la cual el resultado puede relacionarse con una referencia mediante una cadena ininterrumpida y documentada de calibraciones, cada una de las cuales contribuye a la incertidumbre de medida

\section{DESCRIPCIÓN GENERAL DE UN VELOCÍMETRO}

El primer paso para desarrollar un método de calibración es conocer de forma general el instrumento por calibrar. De esta forma es posible tener una mejor comprensión de la medición a la luz de la forma en que el equipo a calibrar opera. Para este caso particular, el equipo sujeto a calibración es un velocímetro en su función de medición de distancia.

Los velocímetros son equipos fijos que pueden emplearse para verificar el estado de

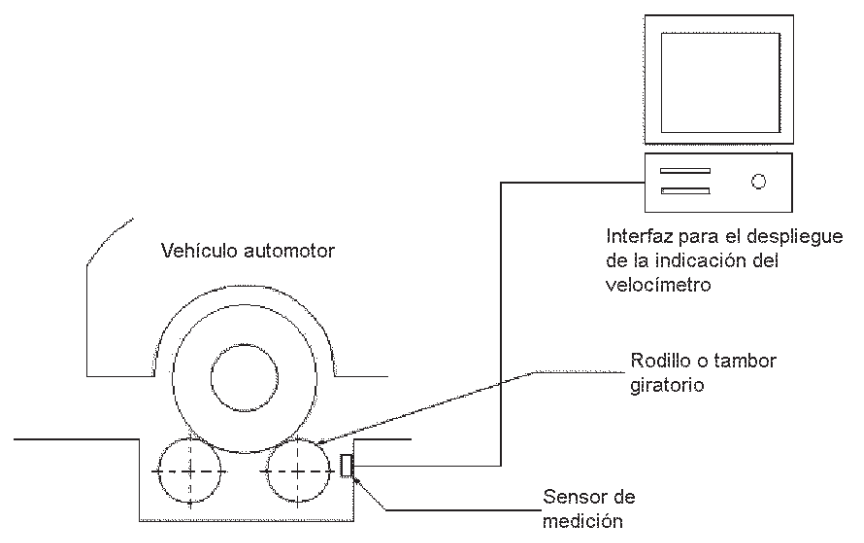

Figura 1. Diagrama esquemático de un velocímetro.

Fuente: (El autor). 
los odómetros o taxímetros de los vehículos en las líneas de inspección o talleres de servicio mecánico. Un velocímetro usualmente se emplea para varias funciones entre las que está la prueba estacionaria realizada para medir la distancia recorrida por las ruedas del vehículo.

La Figura 1 muestra el diagrama esquemático de un velocímetro típico. Existen diversos diseños dependiendo del fabricante, pero en términos generales están conformados por un rodillo o tambor giratorio, un sensor de pulsos (usualmente magnético) y una interfaz que permite desplegar las mediciones.

Para llevar a cabo una prueba de inspección con el velocímetro, una de las llantas del eje que posee tracción en el vehículo por inspeccionar se monta sobre los rodillos de manera que la rotación de la llanta sea transmitida al rodillo cuando la llanta gira. Mediante un sistema de adquisición de datos la señal es transferida a un programa computacional en el cual se determina la distancia recorrida que es desplegada directamente en la interfaz.

\section{PRINCIPIO DE OPERACIÓN DE UN VELOCÍMETRO}

En un velocímetro, el principio de operación consiste en asociar un número de revoluciones aplicadas al rodillo con una distancia que se despliega en la interfaz del mismo. Para ello, internamente puede tomar como variable de entrada la longitud de contorno o el diámetro del rodillo, dependiendo del fabricante.

En uno de los diseños instrumentales, el rodillo tiene una rueda dentada acoplada a uno de sus extremos y en un punto fijo se tiene un sensor electrónico con sensibilidad magnética que reacciona cada vez que uno de los dientes de la rueda dentada interactúa con dicho sensor. El conjunto de interacciones con el sensor generan una señal pulsante que es captada por el sistema de adquisición de datos y procesada en un programa computacional con el fin de determinar y desplegar la distancia recorrida. El velocímetro utilizado para la validación tenía este tipo de diseño instrumental.

Otro de los diseños posee una serie de imanes en la periferia del tambor de manera que cada vez que uno de los imanes interactúa con el sensor se genera un pulso eléctrico que se procesa de forma similar a la señal pulsante descrita antes.

En todos los casos, estos sistemas se basan en convertir el desplazamiento angular del rodillo en una distancia recorrida a partir de la longitud de circunferencia u otra característica dimensional del tambor.

Para calibrar un velocímetro se compara la distancia indicada en la interfaz del equipo sujeto a calibración con la distancia patrón, determinada a partir del número de ciclos y del valor de la circunferencia, del tambor del velocímetro.

\section{MÉTODO DE CALIBRACIÓN}

La calibración se realiza por un método indirecto en el que se compara la lectura del velocímetro contra una distancia patrón. El patrón corresponde a un sistema de medición basado en la longitud de contorno del tambor y un número asociado de ciclos.

La longitud de contorno del tambor se determina a partir de una adecuada caracterización geométrica del rodillo. Luego, el producto del mejor estimador de esta longitud por el mejor estimador del número de ciclos asociados al punto de calibración conforma la distancia patrón.

Para atender este método desde el punto de vista instrumental se necesitan básicamente dos equipos: el primero es un calibrador vernier con capacidad de medir el diámetro del tambor y el segundo es un tacómetro óptico para contar el número de ciclos durante la calibración.

El calibrador vernier utilizado debe estar calibrado y sujeto a control metrológico. Sus mandíbulas deben tener una longitud mayor al radio del rodillo y su capacidad de medición debe ser al menos igual al diámetro del rodillo.

El velocímetro utilizado en la validación tenía un rodillo con un diámetro nominal de $200 \mathrm{~mm}$, por esta razón las mandíbulas del calibrador vernier utilizado debían medir más de $100 \mathrm{~mm}$, de lo contrario no sería geométricamente posible medir el diámetro del rodillo. El calibrador vernier utilizado tenía mandíbulas de $125 \mathrm{~mm}$ de longitud y una capacidad de medición de $1000 \mathrm{~mm}$. 
Para realizar la calibración del velocímetro se monta un vehículo sobre el rodillo o tambor y se hacen girar las llantas del vehículo hasta alcanzar la distancia requerida en cada punto de calibración. La indicación del velocímetro es registrada y comparada contra la distancia patrón dada por el sistema de medición. El deslizamiento entre la llanta y el tambor no afecta las indicaciones del velocímetro durante la calibración dado que lo que realmente interesa es la cantidad de revoluciones del tambor giratorio y no la distancia recorrida por el vehículo automotor.

Vale la pena aclarar que el usuario del velocímetro sí debería considerar el deslizamiento entre la llanta y el rodillo como parte de las fuentes de error cuando realice la prueba de inspección del vehículo. Existe un parámetro denominado coeficiente de rodadura con el cual se puede relacionar la distancia real recorrida por la llanta de un vehículo y la distancia recorrida por un punto arbitrario ubicado sobre el tambor. El coeficiente de rodadura es función de la velocidad de la llanta, de la presión del neumático, del peso, del coeficiente de fricción estático entre las llantas y el tambor, además de otras características de las llantas y de la superficie de contacto. Para efectos de la calibración, el coeficiente de rodadura es intrascendente, en vista de que el mismo está relacionado más con el vehículo y no con el equipo sujeto a calibración.

\section{DEFINICIÓN DEL MENSURANDO}

El mensurando corresponde al error de indicación del velocímetro en su función de medición de distancia para cada uno de los puntos de calibración. Esto es la diferencia entre la indicación del velocímetro y la distancia patrón, esta diferencia tiene signo. Un error positivo implica que el equipo está midiendo por encima del valor verdadero, un error negativo implica que la medición es menor al valor verdadero.

Es importante aclarar que el mensurando está estrictamente relacionado con la indicación de distancia del velocímetro y no con la distancia recorrida por el vehículo que se montará sobre el velocímetro. Puede haber deslizamiento entre las llantas del vehículo y el rodillo.

\section{MODELO DE MEDICIÓN}

Físicamente un modelo consiste en un conjunto de suposiciones sobre el propio mensurando y las variables físicas relevantes en la medición, que pueden incluir entre muchos otros, aspectos geométricos. Una medición, desde el punto de vista físico, por simple que sea, tiene asociado un modelo que solo se aproxima al proceso real.

Particularmente, en un velocímetro la suposición más relevante es considerar al tambor giratorio como un cilindro de diámetro constante. Bajo esta suposición, la longitud de contorno del tambor queda reducida a una longitud de circunferencia basada en el mejor estimador del diámetro, el cual es determinado a partir de un muestreo representativo.

El error de indicación del velocímetro en cada uno de los j-ésimos puntos de calibración se define como:

$e_{j}=I_{j}-P_{j}$

donde:

$e_{j}$ : error de indicación del velocímetro en el j-ésimo punto de calibración, $\mathrm{m}$

$I_{j:} \quad$ indicación del velocímetro en el j-ésmio punto de calibración, $m$

$P_{j}$ : patrón de distancia en el j-ésimo punto de calibración, $m$

El mejor estimador de la indicación del velocímetro en el $j$-ésimo punto de calibración está dado por:

$$
I_{j}=\bar{I}_{j}+\delta I_{r e s}+\delta I_{r e p, j}
$$

donde:

$I_{j}$ : $\quad$ indicación del velocímetro en el $j$-ésimo punto de calibración, $\mathrm{m}$

$\overline{I_{j}}$ : promedio de las indicaciones del velocímetro en el $j$-ésimo punto de calibración, $m$ 
$\delta I_{\text {res }}: \quad$ corrección debida a la resolución del velocímetro, $\mathrm{m}$

$\delta I_{\text {rep }, j}$ : corrección debida a la dispersión de las indicaciones del velocímetro en condiciones de repetibilidad en el $\mathrm{j}$ ésimo punto de calibración, $\mathrm{m}$

En la ecuación (2), las correcciones $\delta I_{\text {res }}$ y $\delta I_{\text {rep, } j}$ tienen esperanza matemática cero pero aportan incertidumbre a la medición. Por lo tanto, la ecuación (1) queda reducida a:

$I_{j}=\overline{I_{j}}$

La distancia patrón en el $j$-ésimo punto de calibración se puede determinar como el producto del mejor estimador de la longitud de contorno del tambor por el mejor estimador del número de ciclos asociados al punto de calibración como sigue:

$P_{j}=C \cdot N_{j}$

donde:

$P_{j}$ : distancia patrón en el $j$-ésimo punto de calibración, $m$

$C$ : mejor estimador de la longitud de contorno del tambor, $\mathrm{m}$

$N_{j}$ : mejor estimador del número de ciclos asociados al punto de calibración

El mejor estimador de la longitud de contorno está dado por:

$$
C=\frac{\pi \cdot D}{1000}
$$

donde:

$C$ : mejor estimador de la longitud de contorno del tambor, $m$

$D$ : mejor estimador del diámetro del tambor, $\mathrm{mm}$

El divisor 1000 que aparece en el denominador del segundo término de la ecuación (5) existe para atender el cambio de unidades del diámetro de milímetros a metros. El mejor estimador del diámetro del tambor está dado por:

$D=\bar{D}+\delta D_{E M P}+\delta D_{\Delta T}+\delta D_{\text {rep }}+\delta D_{\text {tec }}$

donde:

D: mejor estimador del diámetro del tambor, $\mathrm{mm}$

$\bar{D}: \quad$ media aritmética de las mediciones del diámetro de contorno del tambor, $\mathrm{mm}$

$\delta D_{E M P}:$ corrección debida al error máximo permisible del calibrador vernier, $\mathrm{mm}$

$\delta D_{\Delta T}$ : corrección debida a los cambios de temperatura, $\mathrm{mm}$

$\delta D_{\text {rep }}$ : corrección debida a la dispersión de los datos del diámetro del tambor en condiciones de repetibilidad, $\mathrm{mm}$

$\delta D_{\text {tec }}$ : corrección debida a la variabilidad estadística por las mediciones entre analistas, $\mathrm{mm}$

En la ecuación (6), las correcciones $\delta D_{E M P}$, $\delta D_{\Delta T}, \delta D_{\text {rep }}$ y $\delta D_{\text {tec }}$ tienen esperanza matemática pero aportan incertidumbre a la medición. Por lo tanto, la ecuación (6) puede reducirse a:

$$
D=\bar{D}
$$

El mejor estimador del número de ciclos asociados al punto de calibración está dado por:

$N_{j}=\overline{N_{j}}+\delta N_{r e s}$

donde:

$N_{j}$ : $\quad$ mejor estimador del número de ciclos asociados al punto de calibración

$\overline{N_{j}}$ : número de ciclos asociados al punto de calibración indicado por el tacómetro en el $j$-ésimo punto y corresponde a un valor constante. 
$\begin{aligned} \delta N_{\text {res }}: & \begin{array}{l}\text { corrección debida al redondeo del } \\ \text { tacómetro. }\end{array}\end{aligned}$

En la ecuación (8) la corrección $\delta N_{\text {res }}$ tiene esperanza matemática igual a $1 / 2$, esto se debe a la forma en que redondea automáticamente un tacómetro. Esto dado que el tacómetro sólo puede contar el número de ciclos completados. Si por alguna razón se tiene un ciclo que ha sido completado en más de la mitad, el tacómetro no es capaz de contabilizarlo porque sólo mide el número entero de ciclos redondeado hacia abajo. Por lo tanto, el mejor tratamiento estadístico que se le puede dar a $\delta N_{\text {res }}$ es el de una distribución probabilística con esperanza matemática $1 / 2$ para compensar el posible sesgo debido a la resolución del tacómetro. De acuerdo con este razonamiento la ecuación (8) queda reducida a:

$$
N_{j}=\overline{N_{j}}+\frac{1}{2}
$$

donde:

$N_{j}$ : mejor estimador del número de ciclos asociados al punto de calibración

$\overline{N_{j}}$ : número de ciclos asociados al punto de calibración indicado por el tacómetro en el $j$-ésimo punto y corresponde a un valor constante.

Si se sustituye (7) en (5) y luego esta misma ecuación se sustituye en (4) junto con (8) la longitud patrón queda expresada como:

$$
P_{j}=\frac{\pi \cdot \bar{D} \cdot\left(\overline{N_{j}}+\frac{1}{2}\right)}{1000}
$$

El modelo de medición para el error de indicación del velocímetro en cada uno de los $j$-ésimos puntos de calibración se obtiene sustituyendo (10) y (3) en (1) y se obtiene:

$$
e_{j}=\bar{I}_{j}-\frac{\pi \cdot \bar{D} \cdot\left(\overline{N_{j}}+\frac{1}{2}\right)}{1000}
$$

donde:

$e_{j}: \quad$ error de indicación del velocímetro en el j-ésimo punto de calibración, $\mathrm{m}$

$\overline{I_{j}}:$ promedio de las indicaciones del velocímetro en el j-ésimo punto de calibración, m

$\bar{D}$ : media aritmética de las mediciones del diámetro de contorno del tambor, $\mathrm{mm}$

$\overline{N_{j}}:$ número de ciclos asociados al punto de calibración indicado por el tacómetro en el j-ésimo punto de calibración.

\section{PROCEDIMIENTO DE MEDICIÓN}

La calibración de un velocímetro se realiza en dos etapas: la caracterización del rodillo y la medición de distancia.

En la etapa de caracterización del rodillo se realizan diez mediciones del diámetro del tambor con el calibrador vernier de la siguiente forma: se realizan cinco mediciones a lo largo del rodillo en una posición angular específica, luego, se gira el rodillo aproximadamente un cuarto de vuelta y se realizan cinco mediciones más a lo largo de la longitud del rodillo en esta nueva posición. Esta forma de realizar las mediciones mitiga el efecto de las posibles deformidades geométricas del rodillo.

Para la etapa de medición de distancia se calcula el número de revoluciones asociadas al $j$-ésimo punto de calibración a partir de la siguiente relación, la cual es constante para cada $j$-ésimo punto:

$$
\overline{N_{j}}=\frac{1000 \cdot X_{j}}{\pi \cdot \bar{D}}
$$

donde:

$\overline{N_{j}}$ : número de ciclos asociados al punto de calibración indicado por el tacómetro en el j-ésimo punto de calibración. 
$X_{j}$ : $\quad j$-ésimo punto nominal de calibración, $\mathrm{m}$

$\bar{D}$ : media aritmética de las mediciones del diámetro de contorno del tambor, $\mathrm{mm}$

El número de revoluciones $\overline{N_{j}}$ obtenido de la ecuación (12) es redondeado al entero más cercano y es un valor único para cada punto de calibración. Como se puede observar, es función del diámetro promedio del rodillo el cual debe ser determinado previamente.

Una vez determinado el número de ciclos en el punto de calibración, se procede a girar el tambor del velocímetro con ayuda del vehículo hasta completar el número de ciclos respectivo. Este proceso de medición se repite tres veces por cada punto de calibración de tal forma que la indicación del velocímetro quede determinada a partir del promedio de tres indicaciones en condiciones de repetibilidad en cada punto de calibración.

No es recomendable realizar más de tres mediciones por punto de calibración ya que esto provocaría que el procedimiento de calibración sea insostenible desde el punto de vista de costo y recursos para el laboratorio de calibración.

\section{ESTIMACIÓN DE LA INCERTIDUMBRE DE LA MEDICIÓN}

Se identificaron y analizaron las siguientes fuentes de incertidumbre en la medición, derivadas a partir del modelo de medición descrito por la ecuación (11):

- Incertidumbre por resolución de las indicaciones del velocímetro.

- Incertidumbre por redondeo del número de ciclos al entero inferior debido a la resolución del tacómetro.

- Incertidumbre por el error máximo permitido para el calibrador vernier.

- Incertidumbre por la diferencia de temperatura entre el calibrador vernier y el tambor del velocímetro.

- Incertidumbre por falta de repetibilidad de las mediciones del diámetro del tambor.

- Incertidumbre por la variabilidad estadística entre los técnicos que realizan la medición del diámetro del tambor.
- Incertidumbre por falta de repetibilidad de las indicaciones del velocímetro.

Seguidamente, se presenta la evaluación de la incertidumbre estándar de cada fuente. Luego, se muestran los coeficientes de sensibilidad y por último se presenta la incertidumbre expandida de la medición. Esta metodología está de acuerdo con los lineamientos de la Guide to the expression of uncertainty in measurement. (BIPM, IEC, IFCC, ISO, IUPAC, IUPAP \& OIML, 1995).

\subsection{Evaluación de las fuentes de incertidumbre estándar}

\subsubsection{Incertidumbre por resolución de las indicaciones del velocímetro}

La incertidumbre estándar asociada a la resolución de las indicaciones en el velocímetro, considerando que la resolución sigue una distribución rectangular simétrica, se determina según la ecuación (13).

$u\left(\delta I_{\text {res }}\right)=\frac{d}{2 \sqrt{3}}$

Donde:

$u\left(\delta I_{\text {res }}\right): \quad$ Incertidumbre estándar por resolución del velocímetro, $m$

$d_{e}: \quad$ Resolución de la pantalla de indicación del velocímetro, $m$

Se debe tener especial cuidado con la resolución ya que su incertidumbre debe basarse en el umbral de discriminación del equipo de medición según la mínima sensibilidad que tiene ante el movimiento rotatorio del rodillo. Por ejemplo, algunos velocímetros despliegan en sus pantallas valores con una expansión decimal de hasta $0,01 \mathrm{~m}$; sin embargo, el umbral de discriminación puede ser apenas de $0,05 \mathrm{~m}$. En tales casos el valor de $d_{e}$ corresponde a 0,05 $\mathrm{m}$ y no a $0,01 \mathrm{~m}$. 


\subsubsection{Incertidumbre por redondeo del número de ciclos al entero inferior debido a la resolución del tacómetro.}

La incertidumbre estándar asociada al redondeo del número de ciclos al entero inferior debido a la resolución del tacómetro sigue una distribución rectangular y se determina según la ecuación (14).

$$
u\left(\delta N_{r e s}\right)=\frac{d_{N}}{2 \sqrt{3}}
$$

Donde:

$u\left(\delta N_{\text {res }}\right): \quad$ Incertidumbre estándar por resolución en el tacómetro.

$d_{N}$ : Resolución del tacómetro.

Dado que el número de revoluciones es un número entero, la resolución $\mathrm{d}_{N}$ siempre corresponderá a una unidad.

\subsubsection{Incertidumbre por el error máximo permitido para el calibrador vernier.}

La incertidumbre estándar asociada al error máximo permitido para el calibrador vernier se determina de acuerdo con la siguiente ecuación:

$$
u\left(\delta D_{E M T}\right)=\frac{E M P}{\sqrt{3}}
$$

donde:

$u\left(\delta D_{E M T}\right): \quad$ incertidumbre estándar por exactitud del calibrador vernier, $\mathrm{mm}$.

$E M P$ : error máximo permitido para el calibrador vernier, $\mathrm{mm}$.

El error máximo permitido para el calibrador vernier se declara y se debe tener bajo control metrológico con base en la información y los controles que se dispongan para el instrumento utilizado como patrón. Para este trabajo particular se declaró el error máximo tolerado $E M T$ de $\pm 0,03 \mathrm{~mm}$. Para la verificación del cumplimiento de esta especificación se utilizó la siguiente desigualdad:

$\left|e v_{j}\right|+\left|U_{P}\right| \leq E M P$

donde:

$e v_{j}$ : $\quad$ error de indicación del vernier en el $j$ ésimo punto de calibración, $m$

$U_{P}$ : incertidumbre por trazabilidad reportada por el laboratorio de calibración usualmente reportada al $95 \%$ de confianza, $m$.

Para el vernier, las incertidumbres debidas al desgaste de las mandíbulas, la presencia de suciedad y la deriva entre calibraciones sucesivas, por la experiencia del autor, fueron consideradas en una única incertidumbre, asociada al error máximo permisible $(E M P)$. La elección del valor del $E M P$ depende de la resolución del vernier, en este caso se utilizó un instrumento digital con una resolución de $0,01 \mathrm{~mm}$.

Independientemente del valor declarado para $E M P$, en la medida de lo posible se recomienda llevar controles tales como cartas de control, comprobaciones intermedias o cualquier otro mecanismo para mantener este equipo bajo control metrológico.

Otro enfoque alternativo, que no se usó en este caso, es considerar en forma independiente las incertidumbres para el calibrador vernier debidas a trazabilidad, error de indicación y deriva entre calibraciones sucesivas y no considerar la incertidumbre por error máximo tolerado.

Es innecesario considerar la incertidumbre por resolución del calibrador vernier como una posible fuente de incertidumbre, ya que la misma debería estar incluida como parte de las incertidumbres reportadas en el certificado de calibración del vernier.

8.1.4 Incertidumbre por la diferencia de temperatura entre el calibrador vernier y el tambor del velocímetro

Se registraron las condiciones ambientales bajo las cuales se llevaron a cabo las calibraciones 
de velocímetros por un lapso de dos años. Todas las temperaturas registradas estuvieron entre los $14{ }^{\circ} \mathrm{C}$ y $34{ }^{\circ} \mathrm{C}$, por lo tanto, la temperatura se puede considerar como $24^{\circ} \mathrm{C} \pm 10{ }^{\circ} \mathrm{C}$.

Se consideró que las variaciones de temperatura no afectan la calidad de las indicaciones en interfaz del software aunque sí podrán afectar la longitud de circunferencia del rodillo. Se supondrá que tanto el rodillo como el vernier están fabricados en acero con un coeficiente de expansión térmica lineal de $(11,5 \pm 2) \cdot 10^{-6} 1 / \mathrm{K}^{1}$.

La incertidumbre debida a los cambios de temperatura se debe a que la temperatura del calibrador vernier no necesariamente es igual a la del rodillo sujeto a calibración, por esta razón existe una incertidumbre asociada. Si se considera que esta diferencia de temperaturas nunca será mayor a la diferencia entre la mayor y la menor temperatura ambiental, la incertidumbre estándar se puede estimar considerando que sigue una distribución rectangular como sigue:

$u\left(\delta D_{\Delta T}\right)=\frac{\alpha \cdot \Delta T \cdot \bar{D}}{\sqrt{3}}$

donde:

$u\left(\delta D_{\Delta T}\right): \quad$ incertidumbre estándar por las variaciones de la temperatura entre el calibrador vernier y el rodillo del velocímetro, $\mathrm{mm}$.

$\alpha: \quad$ coeficiente de expansión térmica del acero en $(1 / \mathrm{K})$, considerado como $(11,5 \pm 2) \cdot 10^{-6} 1 / \mathrm{K}$.

$\Delta T: \quad$ variación de temperatura entre el calibrador vernier y el rodillo del velocímetro: $20{ }^{\circ} \mathrm{C}$

8.1.5 Incertidumbre por falta de repetibilidad de las mediciones del diámetro del tambor.

Considerando que las mediciones del diámetro del tambor medidas en condiciones de repetibilidad siguen una distribución normal y que se realizan diez mediciones tal como se describió anteriormente, la incertidumbre por falta de repetibilidad de estas mediciones se determina a partir de la desviación estándar experimental de la media, definida como:

$u\left(\delta D_{r e p}\right)=\frac{s(D)}{\sqrt{10}}$

donde:

$u\left(\delta D_{\text {rep }}\right)$ : incertidumbre estándar por repetibilidad de las mediciones del diámetro del tambor, $\mathrm{mm}$.

$s(D): \quad$ desviación estándar muestral de las mediciones del diámetro, $\mathrm{mm}$.

8.1.6 Incertidumbre por la variabilidad estadística entre los técnicos que realizan la medición del diámetro del tambor

Esta incertidumbre fue determinada en el proceso de validación y corresponde a un valor constante. Se obtuvo a partir de la raíz cuadrada de la varianza entre grupos atendiendo las indicaciones de la norma INTE-ISO 5725-2:2006, Exactitud (veracidad y precisión) de resultados y métodos de precisión - Parte 2: método básico para la determinación de la repetibilidad y la reproducibilidad de un método de medición normalizado (INTECO, 2006).

La ecuación que permite determinar esta incertidumbre está dada por:

$u\left(\delta D_{\text {tec }}\right)=\sqrt{\frac{s_{b}^{2}-s_{w}^{2}}{n}}$

donde:

$u\left(\delta D_{\text {tec }}\right)$ : incertidumbre estándar debida a las variaciones entre analistas, $\mathrm{mm}$

$s_{b}^{2}: \quad$ varianza entre grupos, $\mathrm{mm}^{2}$

$\begin{array}{ll}S_{w}^{2:} & \text { varianza dentro de grupos, } \mathrm{mm}^{2} \\ n: & \text { número de grupos }\end{array}$ 
Para nuestro caso en particular, el valor de esta incertidumbre estándar es de $0,113 \mathrm{~mm}$ y fue determinada a partir de un número de grupos $n=8$ en el proceso de validación.

\subsubsection{Incertidumbre por falta de repetibilidad de las indicaciones del velocímetro}

La incertidumbre estándar por falta de repetibilidad de las indicaciones del velocímetro se estima como la desviación estándar experimental de la media de las tres mediciones realizadas en condiciones de repetibilidad.

$$
u\left(\delta I_{r e p}\right)=\frac{s(I)}{\sqrt{3}}
$$

donde:

$u\left(\delta I_{\text {rep }}\right):$ incertidumbre estándar debida a la falta de repetibilidad de las indicaciones del velocímetro, $\mathrm{m}$

$\mathrm{s}(I)$ desviación estándar muestral de las indicaciones del velocímetro, $\mathrm{m}$

\subsection{Coeficientes de sensibilidad}

Los coeficientes de sensibilidad son valores que al multiplicarse por las incertidumbres estándar respectivas dan como resultado las contribuciones directas de estas incertidumbres en el mensurando de la medición. Matemáticamente se definen como la derivada parcial de cada una de las variables que conforman el modelo de medida con respecto al mensurando. A continuación se presentan los coeficientes de sensibilidad de cada una de las variables presentadas en la ecuación (11).

El coeficiente de sensibilidad para las incertidumbres asociadas al diámetro del tambor $C S_{D j}$ en el j-ésimo punto de calibración está dado por:
$C S_{D j}=\frac{\partial e_{j}}{\partial D}=\frac{-\pi \cdot\left(\overline{N_{j}}+\frac{1}{2}\right)}{1000}$

El coeficiente de sensibilidad para las incertidumbres asociadas al número de ciclos del tambor $C S_{N}$ es independiente del número de ciclos y está dado por:

$C S_{N}=\frac{\partial e_{j}}{\partial N}=\frac{-\pi \cdot \bar{D}}{1000}$

El coeficiente de sensibilidad de las incertidumbres asociadas a la indicación del velocímetro $C S_{I j}$ es unitario y adimensional.

$C S_{I j}=\frac{\partial e_{j}}{\partial I_{j}}=1$

\subsection{Incertidumbre estándar combinada}

La incertidumbre estándar combinada para cada j-ésimo punto de calibración se obtiene a partir de la Ley de Propagación de Incertidumbres. Las contribuciones son el resultado del producto de cada incertidumbre por su respectivo coeficiente de sensibilidad, ecuación (24)

$u_{c}\left(e_{j}\right)$ incertidumbre estándar combinada del error de indicación del velocímetro, $\mathrm{m}$

\subsection{Grados de libertad efectivos}

Con el fin de determinar el factor de cobertura, la Guide to the expression of uncertainty in measurements (BIPM, IEC, IFCC, ISO, IUPAC, IUPAP \& OIML, 1995) propone la

$u_{C}\left(e_{j}\right)=\sqrt{u^{2}\left(\delta I_{r e s}\right)+u^{2}\left(\delta I_{r e p}\right)+C S_{D j}^{2} \cdot\left[u^{2}\left(\delta D_{E M T}\right)+u^{2}\left(\delta D_{\Delta T}\right)+u^{2}\left(\delta D_{r e p}\right)+u^{2}\left(\delta D_{\text {tec }}\right)\right]+C S_{N}^{S} \cdot u^{2}\left(\delta N_{r e s}\right)}$ 
ecuación de Welch-Satterhwaite. A cada una de las incertidumbres se le asignó un número efectivo de grados de libertad.

Para las incertidumbres asociadas a la resolución de las indicaciones del velocímetro, redondeo del tacómetro y el error máximo permitido del vernier, se consideró que el número de grados de libertad es infinito. La incertidumbre por repetibilidad de la medición del diámetro se consideró en nueve grados de libertad, uno menos que el número de mediciones realizadas. En forma análoga, la incertidumbre por las variaciones entre técnicos en la medición del diámetro del tambor se consideró en siete grados de libertad y la incertidumbre por repetibilidad de las mediciones del velocímetro se consideró en dos grados de libertad.

Para determinar el número de grados de libertad de la corrección por variaciones de temperatura se procedió de la siguiente forma:

$v \cong \frac{1}{2} \cdot\left(\frac{\Delta u}{u}\right)^{-2}=\frac{1}{2} \cdot\left(\frac{\Delta \alpha}{\alpha}\right)^{-2}=\frac{1}{2} \cdot\left(\frac{2 \times 10^{-6}}{11,5 \times 10^{-6}}\right)^{-2}=16,53$

Bajo estas consideraciones, el número efectivo de grados de libertad está dado por la ecuación (26):

donde:

$v_{e f}: \quad$ grados efectivos de libertad de la incertidumbre estándar combinada

$u_{c}\left(e_{j}\right)$ incertidumbre estándar combinada del error de indicación del velocímetro, $\mathrm{m}$

\subsection{Incertidumbre expandida}

La incertidumbre expandida del error de indicación del velocímetro $U_{p j}$, se determina según la ecuación:

$$
U_{P j}=k_{P} \cdot u_{C}\left(e_{j}\right)
$$

donde:

$U_{p j}$ : incertidumbre expandida de medición, $\mathrm{m}$

$k_{p}$ : factor de cobertura para un nivel de confianza del 95,45\% obtenido a partir del número efectivo de grados de libertad y la distribución t-Student

$u_{c}\left(e_{j}\right)$ : incertidumbre estándar combinada del error de indicación del velocímetro, $\mathrm{m}$

Se considera que la incertidumbre expandida sigue una distribución t-Student con el número de grados de libertad calculados de la ecuación (26) y el factor de cobertura respectivo. La incertidumbre expandida debe ser reportada con dos cifras significativas y el error de indicación con un número de cifras tal que sea coherente con la incertidumbre.

\section{VALIDACIÓN DEL MÉTODO}

La validación es un proceso por medio del cual se aporta evidencia objetiva para demostrar que el método de calibración es apto para su uso previsto. Dicho uso corresponde a calibrar velocímetros y determinar su error de indicación para verificar la conformidad respecto a una tolerancia establecida. Se tiene como meta que la incertidumbre del método, a un nivel de confianza del 95,45\%, no sea mayor al 0,22\% de la indicación de distancia del velocímetro.

Con el fin de demostrar estadísticamente que el método puede atender este requisito metrológico, se procedió a realizar un diseño de experimentos en el cual se determina la mínima incertidumbre del método de calibración. Si esta incertidumbre cumple con no ser mayor al 0,22\% de la indicación del velocímetro, el método se considera apto para su uso previsto.

$$
v_{e f}=\frac{u_{C}^{4}\left(e_{j}\right)}{\frac{u^{4}\left(\delta I_{r e p}\right)}{2}+\frac{C S_{D j}^{4} \cdot u^{4}\left(\delta D_{r e p}\right)}{9}+\frac{C S_{D j}^{4} \cdot u^{4}\left(\delta D_{\Delta T}\right)}{16,53}+\frac{C S_{D j}^{4} \cdot u^{4}\left(\delta D_{t e c}\right)}{7}}
$$


Para realizar las pruebas de validación se empleó un velocímetro con una resolución de 1 m. Fue calibrado en forma completa por cuatro técnicos por el método descrito anteriormente. Cada técnico calibró el equipo dos veces en días distintos con el fin de evaluar la reproducibilidad hasta completar ocho calibraciones. Cada calibración se considera una muestra independiente y cada una será tratada como un tratamiento independiente. La calibración se llevó a cabo en cuatro puntos de calibración a saber: 1000 m; 1500 m; 2000 m y 2500 m.

Con todos los datos obtenidos se prosiguió con una metodología de validación a través de las siguientes etapas:

1) Análisis de la precisión de la medición del diámetro del rodillo.

2) Análisis de la precisión de las indicaciones del velocímetro.

3) Estimación de la incertidumbre estándar combinada.

4) Estimación de la incertidumbre expandida a un nivel de confianza del 95,45\%.

\subsection{Análisis de la precisión de la medición del diámetro del rodillo}

En el Cuadro No. 1 se muestran los resultados de cada uno de los ocho tratamientos con su respectiva media y desviación estándar muestral.

Con el fin de evaluar si alguno de los tratamientos corresponde a un dato anómalo se realizó una Prueba de Grubbs. Para ello se ordenaron las medias de cada tratamiento en orden ascendente y con base en la mínima y la máxima media del diámetro se determinaron los siguientes estadísticos:

$$
G_{\max }=\frac{D_{\max }-\bar{D}}{s}=\frac{199,719-199,472}{0,1211}=2,04
$$

$$
G_{\min }=\frac{\bar{D}-D_{\min }}{s}=\frac{199,472-199,341}{0,1211}=1,08
$$

donde:

$s: \quad$ es la desviación estándar muestral de las medias de los diámetros de cada muestra, $\mathrm{mm}$

$D_{\text {min }}$ : mínimo de los promedios del diámetro, $\mathrm{mm}$

$D_{\max }$ : máximo de los promedios del diámetro, $\mathrm{mm}$

$\bar{D}$ : media aritmética de las medias de los diámetros, $\mathrm{mm}$

$G_{\text {max }}$ : $\quad$ valor crítico de Grubbs para analizar el valor máximo, $\mathrm{mm}$

$\mathrm{G}_{m i n}$ : valor crítico de Grubbs para analizar el valor mínimo, $\mathrm{mm}$

Dado que el valor crítico de Grubbs al $5 \%$ es de 2,126, se considera que no hay evidencia de que alguna de las medias corresponda a un valor anómalo.

Luego, se realizó una Prueba de Cochran para detectar si alguna de las varianzas es mucho mayor que las demás. Para ello se calculó el siguiente estadístico:

$$
G=\frac{\text { más grande } s_{i}^{2}}{\sum_{i=1}^{8} s_{i}^{2}}=\frac{0,0411}{0,1504}=0,2732
$$

donde:

$G: \quad$ estadístico de Cochran

$s_{i}^{2}: \quad$ i-ésima varianza, en $\mathrm{mm}^{2}$

Como el valor crítico de Cochran es 0,2926 se considera que no hay evidencia al $95 \%$ de significancia de que exista una varianza mayor a las demás.

Seguidamente, se aplicó la técnica del Análisis de Varianza (ANOVA) de un solo factor para determinar si existe una diferencia significativa entre las medias de los ocho tratamientos. Para ello, se busca probar la siguiente hipótesis estadística:

$$
\mathrm{H}_{0}: \mu_{1}=\mu_{1}=\ldots=\mu_{8}
$$

$\mathrm{H}_{1}$ : Al menos dos de las medias de los tratamientos no son iguales 
Los resultados del Análisis de varianza se muestran en el Cuadro 2.

Como el valor crítico de la distribución $\mathrm{F}$ para siete y setenta y dos grados de libertad al $95 \%$ de significancia es 2,14 se deduce de la aplicación de esta técnica que hay evidencia de que existe una diferencia significativa entre las medias. Con el fin de evaluar la variación entre tratamientos y dentro de cada tratamiento se calcularon respectivamente las desviaciones estándar asociadas a la variación interanalista y la repetibilidad en forma como sigue:

$s_{L}=\sqrt{\frac{s_{b}^{2}-s_{w}^{2}}{n}}=\sqrt{\frac{0,1466-0,0188}{10}}=0,113 \mathrm{~mm}$

$s_{r}=\sqrt{s_{w}^{2}}=\sqrt{0,0188}=0,137 \mathrm{~mm}$

donde:

$\mathrm{s}_{L}: \quad$ desviación estándar interanalista, $\mathrm{mm}$

sr: desviación estándar por repetibilidad, $\mathrm{mm}$

$\mathrm{s}_{b}^{2}$ : variación entre grupos o tratamientos, $\mathrm{mm}^{2}$

$\mathrm{S}_{w}{ }^{2}$ : variación dentro del grupo o tratamiento, $\mathrm{mm}^{2}$

\subsection{Análisis de la precisión de las indicaciones del velocímetro}

El análisis de precisión de las indicaciones del velocímetro se realizó para cada uno de los puntos de calibración. Los puntos de calibración corresponden a $1000 \mathrm{~m}, 1500 \mathrm{~m}, 2000 \mathrm{~m}$ y $2500 \mathrm{~m}$. Dado que cada analista tiene un diámetro promedio distinto y por ende un número de ciclos asociado al punto de calibración distinto, se trabajó con el error de indicación dado por (11) en vez de la indicación directa para compensar las variaciones por mediciones del diámetro.

En cada punto de calibración se aplicó Análisis de Varianza en forma similar al Cuadro No. 2. En los cuatro puntos de calibración se concluyó que no hay evidencia al $95 \%$ de confianza de que exista una diferencia significativa entre las medias. Por lo tanto, es razonable calcular para cada punto de calibración una desviación estándar mancomunada.

La desviación estándar mancomunada corresponde a la desviación estándar muestral de los datos que conforman todos los tratamientos tal como si provinieran de una sola muestra. En el Cuadro No. 3 se muestra una síntesis de los resultados respectivos.

Cuadro 1. Datos del diámetro del tambor del velocímetro en mm

$\begin{array}{ccccccccc}\text { Dato } & \mathbf{1} & \mathbf{2} & \mathbf{3} & \mathbf{4} & \mathbf{5} & \mathbf{6} & \mathbf{7} & \mathbf{8} \\ 1 & 199,48 & 199,78 & 199,43 & 199,75 & 199,50 & 199,60 & 199,55 & 199,51 \\ 2 & 199,42 & 199,62 & 199,70 & 199,52 & 199,54 & 199,89 & 199,42 & 199,53 \\ 3 & 199,41 & 199,47 & 199,35 & 199,53 & 199,27 & 199,38 & 199,27 & 199,27 \\ 4 & 199,24 & 199,41 & 199,38 & 199,96 & 199,33 & 199,23 & 199,31 & 199,31 \\ 5 & 199,42 & 199,43 & 199,41 & 199,87 & 199,38 & 199,54 & 199,43 & 199,45 \\ 6 & 199,48 & 199,70 & 199,51 & 199,58 & 199,49 & 199,73 & 199,47 & 199,49 \\ 7 & 199,51 & 199,57 & 199,63 & 199,66 & 199,51 & 199,41 & 199,26 & 199,33 \\ 8 & 199,38 & 199,39 & 199,75 & 199,78 & 199,37 & 199,29 & 199,20 & 199,18 \\ 9 & 199,31 & 199,39 & 199,48 & 199,73 & 199,43 & 199,39 & 199,16 & 199,18 \\ 10 & 199,46 & 199,38 & 199,55 & 199,81 & 199,48 & 199,45 & 199,34 & 199,26 \\ \text { Promedio } & 199,411 & 199,514 & 199,519 & 199,719 & 199,430 & 199,491 & 199,341 & 199,351 \\ \text { Varianza } & 0,0069 & 0,0209 & 0,0187 & 0,0213 & 0,0079 & 0,0411 & 0,0155 & 0,0180 \\ \text { Desviación estándar } & 0,0832 & 0,1446 & 0,1369 & 0,1461 & 0,0889 & 0,2027 & 0,1246 & 0,1341 \\ & & & \text { Fuente: (El autor). } & & & & \end{array}$




\subsection{Estimación de la incertidumbre estándar combinada}

La estimación de la incertidumbre se realizó siguiendo las ecuaciones presentadas en la sección 7. La incertidumbre por repetibilidad del diámetro se estimó a partir de la variación dentro de grupos. La repetibilidad de las indicaciones del velocímetro se determinó a partir de la incertidumbre mancomunada en cada punto de calibración. En los Cuadros del 4 al 7 se presentan los presupuestos de incertidumbre para cada uno de los puntos de calibración: 1000 m, 1500 m, 2000 m y 2500 m.

En la Figura 2 se muestra un diagrama de pesos de las incertidumbres. Se puede observar que la mayor contribución se debe a la variabilidad interanalista en la caracterización del rodillo.

\subsection{Estimación de la incertidumbre expandida a un nivel de confianza del $95,45 \%$}

Para la estimación de la incertidumbre expandida se utilizó la ecuación (27) en cada punto de calibración. En el Cuadro 8 se presentan los resultados de la incertidumbre expandida los cuales siguen una distribución t-Student con el número de grados de libertad dados. La incertidumbre expandida también se presenta en forma porcentual y puede observarse que en todos los casos es menor al $0,22 \%$. Por lo tanto, se considera que el método de calibración es apto para su uso previsto.

Cuadro 2. Análisis de varianza para la medición del diámetro

$\begin{array}{ccccc}\text { Fuente de variación } & \begin{array}{c}\text { Suma de } \\ \text { cuadrados }\end{array} & \begin{array}{c}\text { Grados de } \\ \text { libertad }\end{array} & \begin{array}{c}\text { Cuadrados } \\ \text { medios }\end{array} & \text { Calculada } \\ \text { Tratamientos } & 1,026 & 7 & 0,1466 & 7,80 \\ \text { Error } & 1,354 & 72 & 0,0188 & \\ \text { Total } & 2,380 & 79 & \text { F crítica } & 2,14 \\ & & & & \text { Se rechaza }\end{array}$

Fuente: (El autor).

Cuadro 3. Incertidumbre estándar mancomunada de la variación de las indicaciones del velocímetro

Punto de calibración

(m)

1000

1500

2000

2500
Incertidumbre mancomunada

$(\mathrm{mm})$

0,683

0,871

0,946

0,643

Fuente: (El autor). 
Cuadro 4. Presupuesto de incertidumbres para el punto de calibración de $1000 \mathrm{~m}$

\section{Fuente}

Variaciones de temperatura

Error máximo permisible para el calibrador vernier

Repetibilidad del diámetro

Variabilidad interanalista

Resolución del tacómetro

Resolución del velocímetro

Repetibilidad de las indicaciones del velocímetro

\section{Incertidumbre estándar}

0,026

0,058

0,043

0,113

0,289

0,289

0,371

Incertidumbre estándar combinada

$-5,01$

$-5,01$

$-5,01$

$-0,63$

1,00

1,00

Contribución a la
incertidumbre (m)

Grados de libertad
6,53

$\infty$

9

7

$\infty$

$\infty$

2

21

Fuente: (El autor).

Cuadro 5. Presupuesto de incertidumbres para el punto de calibración de $1500 \mathrm{~m}$

\begin{tabular}{lcccc}
\multicolumn{1}{c}{ Fuente } & $\begin{array}{c}\text { Incertidumbre } \\
\text { estándar }\end{array}$ & $\begin{array}{c}\text { Coeficiente de } \\
\text { sensibilidad }\end{array}$ & $\begin{array}{c}\text { Contribución a la } \\
\text { incertidumbre (m) }\end{array}$ & $\begin{array}{c}\text { Grados de } \\
\text { libertad }\end{array}$ \\
Variaciones de temperatura & 0,026 & $-7,52$ & 0,20 & 16,53 \\
$\begin{array}{l}\text { Error máximo permisible para el } \\
\text { calibrador vernier }\end{array}$ & 0,058 & $-7,52$ & 0,43 & $\infty$ \\
$\begin{array}{l}\text { Repetibilidad } \\
\text { Variabilidad interanalista }\end{array}$ & 0,043 & $-7,52$ & 0,33 & 7 \\
$\begin{array}{l}\text { Resolución del tacómetro } \\
\text { Resolución del velocímetro }\end{array}$ & 0,113 & $-7,52$ & 0,85 & $\infty$ \\
$\begin{array}{l}\text { Repetibilidad de las indicaciones } \\
\text { del velocímetro }\end{array}$ & 0,289 & $-0,63$ & 0,18 & $\infty$ \\
\end{tabular}

Fuente: (El autor). 
Cuadro 6. Presupuesto de incertidumbres para el punto de calibración de $2000 \mathrm{~m}$

\section{Fuente}

Variaciones de temperatura

Error máximo permisible para el calibrador vernier

Repetibilidad

Variabilidad interanalista

Resolución del tacómetro

Resolución del velocímetro

Repetibilidad de las indicaciones del velocímetro

\section{Incertidumbre estándar}

\section{Coeficiente de sensibilidad}

Contribución a la incertidumbre (m)

Grados de libertad

$\begin{array}{cccc}0,026 & -12,53 & 0,33 & 16,53 \\ 0,058 & -12,53 & 0,72 & \infty \\ 0,043 & -12,53 & 0,54 & 9 \\ 0,113 & -12,53 & 1,42 & 7 \\ 0,289 & -0,63 & 0,18 & \infty \\ 0,289 & 1,00 & 0,29 & \infty \\ 0,371 & 1,00 & 0,37 & 2 \\ \text { ncertidumbre estándar combinada } & 1,79 \mathrm{~m} & 17\end{array}$

Fuente: (El autor).

Cuadro 7. Presupuesto de incertidumbres para el punto de calibración de $2500 \mathrm{~m}$

\section{Fuente}

Variaciones de temperatura

Error máximo permisible para el calibrador vernier

Repetibilidad

Variabilidad interanalista

Resolución del tacómetro

Resolución del velocímetro

Repetibilidad de las indicaciones del velocímetro

\section{Incertidumbre estándar}

$$
0,026
$$

0,058

0,043

0,113

0,289

0,289

0,643

Incertidumbre estándar combinada

$$
-12,53
$$$$
-12,53
$$$$
-12,53
$$$$
-12,53
$$$$
-0,63
$$$$
1,00
$$$$
1,00
$$

Contribución a la sensibilidad
Grados de libertad
16,53

$\infty$

9

7

$\infty$

$\infty$

0,64

Fuente: (El autor).

Cuadro 8. Incertidumbre expandida en cada punto de calibración

$\begin{array}{ccccc}\text { Punto de } & \begin{array}{c}\text { Incertidumbre } \\ \text { combinada } \\ \text { ealibración }(m)\end{array} & \begin{array}{c}\text { Grados de } \\ \text { libertad }\end{array} & \begin{array}{c}\text { Factor de cobertura } \\ \text { al 95,45\% de } \\ \text { confianza }\end{array} & \begin{array}{c}\text { Incertidumbre } \\ \text { expandida (m) }\end{array}\end{array}$

\section{Incertidumbre expandida porcentual $(\%)$}

$\begin{array}{llllll}1000 & 0,85 & 21 & 2,13 & 1,8 & 0,18 \\ 1500 & 1,19 & 18 & 2,15 & 2,6 & 0,17 \\ 2000 & 1,51 & 18 & 2,15 & 3,3 & 0,16 \\ 2500 & 1,79 & 17 & 2,16 & 3,9 & 0,15\end{array}$

Fuente: (El autor). 


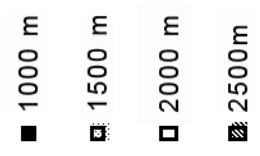

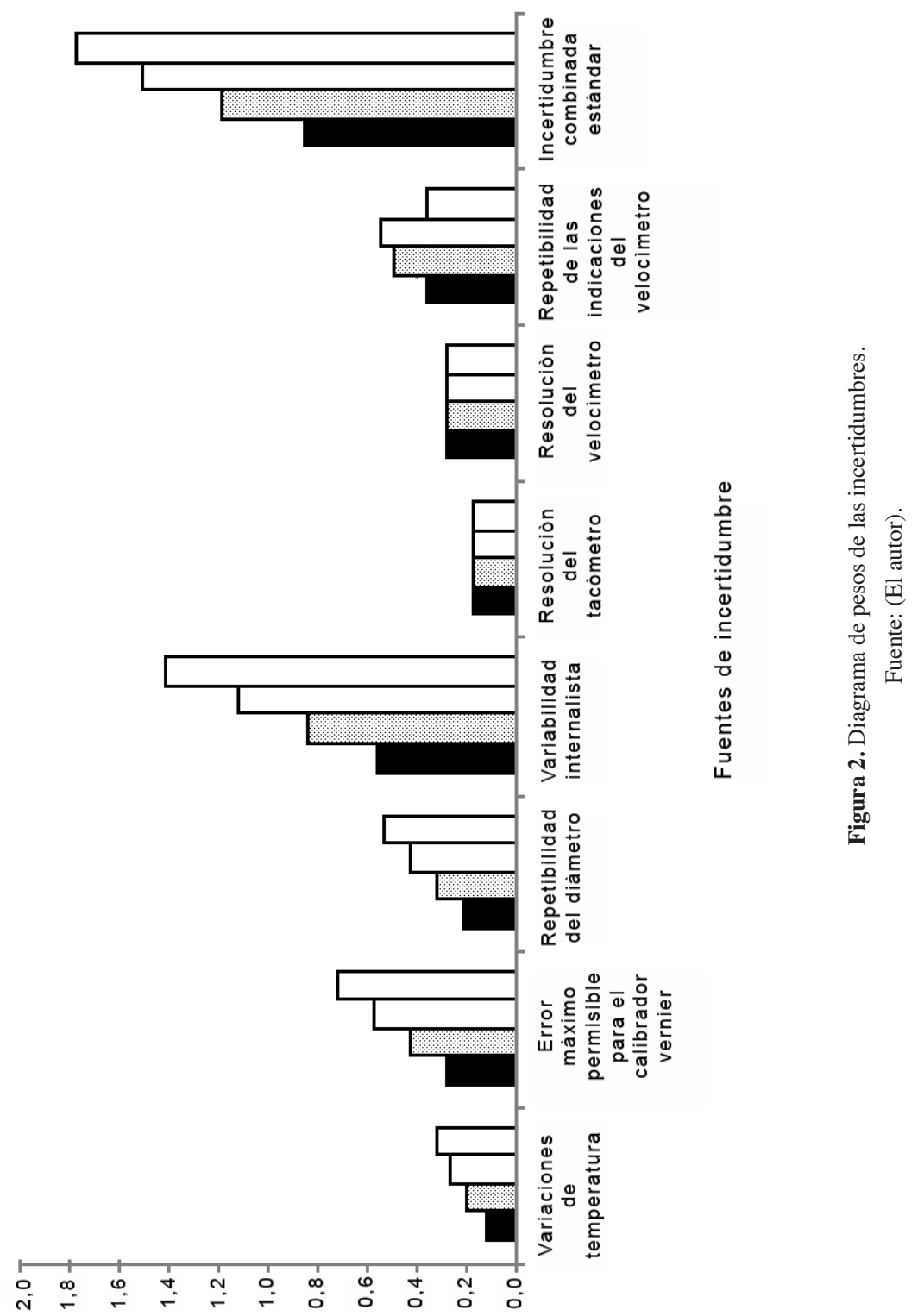




\section{CONCLUSIONES}

1. De la información obtenida en la validación se puede concluir que la incertidumbre con mayor influencia corresponde a las variaciones interanalista. Esta es una oportunidad de mejora que puede solventarse con un mayor entrenamiento del personal técnico que realiza calibraciones. Una reducción en la incertidumbre inter-analista podría permitir determinar que no existe variación significativa entre las medias de los tratamientos permitiendo eliminar la variación interanalista del presupuesto de incertidumbres. Esto reduciría significativamente la incertidumbre.

2. La incertidumbre del método no cumple con los lineamientos del Teorema del Límite Central debido a que el número de grados de libertad de las incertidumbres por repetibilidad de las indicaciones del velocímetro y las variaciones interanalista son números bajos. Un aumento en la cantidad de mediciones de las indicaciones del velocímetro y una reducción en la repetibilidad interanalista podrían ayudar a que el número de grados efectivos de la incertidumbre combinada estándar aumenten y se dé una mejor aplicación del Teorema del Límite Central.

3. Con todo esto se ha logrado desarrollar y validar exitosamente un método de calibración de velocímetros en su función de medición de distancia en el rango que va de $1000 \mathrm{~m}$ a 2500 $m$ con una incertidumbre menor a $0,22 \%$ de la indicación del equipo y con un potencial para mejorar esta incertidumbre a futuro.

4. Con este procedimiento, la Universidad de Costa Rica ha logrado posicionarse en la estructura metrológica de segundo nivel nacional en la calibración de velocímetros con una propuesta oportuna y de alto valor técnico para atender una necesidad metrológica particular.

\section{NOTAS}

1. Véase recomendación en "Uncertainty and dimensional calibration" del "Journal of Research of the National Institute of Standards and Technology", volumen 102, número 6 de noviembre-diciembre 1997.

2. Las actividades fueron realizadas entre el 25 y el 27 de marzo del 2008.

\section{REFERENCIAS BIBLIOGRÁFICAS}

INTECO. (2006). INTE-ISO 5725-2:2006, Exactitud (veracidad y precisión) de resultados y métodos de precisión - Parte 2: método básico para la determinación de la repetibilidad y la reproducibilidad de un método de medición normalizado. Primera edición. San José: Editor.

BIPM, IEC, IFCC, ISO, IUPAC, IUPAP \& OIML. (1995). Guide to the expression of uncertainty in measurement. First edition. International Organization for Standarization: Switzerland.

Dorion, T. \& Stoup, J. (1997). Uncertainty and dimensional calibration. Journal of Research of the National Institute of Standards and Technology, 6(102), 647-676.

Schmid, W. \& Lazos, R. (2000). Guía para estimar la incertidumbre de la medición. México: CENAM.

\section{SOBRE EL AUTOR}

\section{Raziel Farid Sanabria Sandí}

M. Sc. en Ingeniería Mecánica con énfasis en sistemas Térmicos e Hidráulicos de la Universidad de Costa Rica

Investigador del Laboratorio de Metrología, Normalización y Calidad del Instituto de Investigaciones en Ingeniería de la Universidad de Costa Rica

Profesor de la Escuela de Ingeniería Mecánica de la Universidad de Costa Rica

Teléfono: (506) 2511- 5553

Facsímil: (506) 2253- 4614

Apartado postal: 11501- 2060

Correo electrónico: raziel@ inii.ucr.ac.cr 